InIFolPharm

Chronische lymphatische Leukämie

\title{
Chlorambucil plus CD20-Antikörper
}

\author{
Für die Therapie der chronischen \\ lymphatischen Leukämie (CLL) gab \\ es bisher mit Ausnahme von Chlor- \\ ambucil keinen Standard. Die Kom- \\ bination von Chlorambucil mit den \\ CD-20-Antikörpern Rituximab oder \\ GA101 (Obinutuzumab) prüft die \\ Phase-III-Studie CLL11.
}

GA101 ist ein neuer Typ-II-CD20-Antikörper. Die Wirksamkeit von GA101 wird aktuell in der Phase-III-Studie CLL11 bei 781 im Durchschnitt 70 Jahre alten „Slowgo"-Patienten in drei Gruppen untersucht: $20 \%$ erhielten Chlorambucil (6 Zyklen), $40 \%$ GA101 plus Chlorambucil (6 Zyklen), und $40 \%$ Rituximab plus Chlorambucil (6 Zyklen).

Das progressionsfreie Überleben (PFS) der Patienten, die GA101 und Chlorambucil erhalten hatten, war laut Michael Hallek, Köln/Bonn, um 12,1 Monate länger als bei alleiniger Chemotherapie (PFS 23,0 vs. 10,9 Monte; $\mathrm{p}<0,0001$, Hazard Ratio [HR] 0,14) [Goede V et al. J Clin Oncol. 2013;31(suppl):abstr. 7004]. Vielversprechend waren auch die Ergebnisse für die Remissionsqualität (Komplettremissionen [CR] 22,2 vs. $0 \%$ ) und für die Rate nicht nachweisbarer minimaler Resterkrankung (MRD; Blut: 31,1 vs. $0 \%$, Knochenmark: 17,0 vs. $0 \%$ ). Auch die Kombination von Rituximab und Chlorambucil verlängerte im Vergleich zur Chlorambucil-Therapie alleine das PFS signifikant (PFS 15,7 vs. 10,8 Monate; $\mathrm{p}<0,0001 ;$ HR 0,32 ) und erzielte eine höhere Rate an Komplettremissionen (CR 8,3 vs. $0 \%$ ).

Beide Kombinationstherapien führten zu mehr Nebenwirkungen als die Monotherapie. Die häufiger auftretenden Neutropien unter den beiden Kombinationstherapien (GA101: $34 \%$, Rituximab: $25 \%$, Chlorambucil: $15 \%$ ) waren Hallek zufolge nicht mit häufigeren schweren Infektionen verbunden. Friederike Holthausen

Pressekonferenz "Aktuelles vom amerikanischen Krebskongress 2013“, Bonn, 12.06.2013; Veranstalter: Roche

\section{Es gibt Alternativen zu TACE}

\section{Die transarterielle Chemoemboli- sation (TACE) gilt als Standard für die Behandlung von Patienten mit Leberzellkarzinom (HCC) im inter- mediären Stadium. Abhängig von der Tumorgröße und dem Ausmaß des Lymphknotenbefalls bieten sich auch andere Optionen an.}

Die Gruppe der Patienten mit HCC im intermediären Stadium B nach Barcelona Clinic Liver Cancer (BCLC)-Klassifikation ist nicht nur zahlenmäßig groß, sondern auch heterogen, erläuterte Luigi Bolondi, Bologna, Italien. Deshalb empfiehlt er, HCC-Patienten im intermediären Stadium B in Abhängigkeit von definierten Kriterien, z.B. der Leberfunktion (Child Pugh A-C), dem Allgemeinzustand (ECOG PS 0-2) oder dem Vorliegen einer Pfortaderthrombose (PVT) in Subgruppen einzuteilen und für die jeweiligen
Subgruppen eine primäre Therapieempfehlung plus Alternative zu definieren.

Für Patienten im intermediären Stadium mit großer Tumorlast und PVT ist laut Bolondi der orale Tyrosinkinasehemmer Sorafenib (Nexavar ${ }^{\circledR}$ ) der TACE bzw. TARE (transarteriellen Radioembolisation) vorzuziehen. In der Zulassungsstudie SHARP [Llovet JM et al. N Engl J Med. 2008;359(4):378-90] hatte Sorafenib bei Patienten mit mehrheitlich fortgeschrittenem HCC (Child Pugh C) als erste systemische Therapie einen signifikanten Überlebensvorteil gegenüber Placebo (Kontrollarm) erreicht und das relative Sterberisiko um $31 \%$ reduziert (HR 0,69; $\mathrm{p}<0,001)$.

Birgit-Kristin Pohlmann

Satellitensymposium „Bayer Oncology - Today and Tomorrow", 3rd ITLT (Interdisciplinary Treatment of Liver Tumors) Essen, 18.04.2013; Veranstalter: Bayer Pharma

\section{Neuer Antikörper beim multiplen Myelom}

In einer kleinen, randomisierten, offenen Phase-II-Studie wurden Patienten multiplem Myelom, die auf die vorhergehende Therapie nicht ansprachen oder nach Therapie ein Rezidiv entwickelten, mit zwei Dosierungen des monoklonalen Antikörpers Elotuzumab (10 mg/kg und $20 \mathrm{mg} / \mathrm{kg}$ ) in Kombination mit der derzeitigen Standardtherapie Lenalidomid und niedrig dosiertem Dexamethason behandelt. Bei einer Dosierung von $10 \mathrm{mg}$ Elotuzumab/ $\mathrm{kg}$ in dieser Kombination lag das mediane progressionsfreie Überleben in der Langzeitbeobachtung bei 33 Monaten [Facon T et al. EHA Annual Congress 2013, abstr. P764]. Die objektive Ansprechrate (ORR) betrug $92 \%$.

Die häufigsten Nebenwirkungen des Grads 3 und höher waren Lymphopenie (19\%), Neutropenie (18\%), Thrombozytopenie (16\%) und Anämie (14\%).

Aktuell werden aufgrund der viel versprechenden Ergebnisse zwei Phase-IIIStudien zu Elotuzumab mit einer Dosis von $10 \mathrm{mg} / \mathrm{kg}$ bei Patienten mit vorbehandeltem und neu diagnostiziertem multiplem Myelom durchgeführt. red

Nach Informationen von Bristol-Myers Squibb und AbbVie

\section{Neuzulassung für Prostatakarzinom}

Die Europäische Arzneimittel-Agentur (EMA) hat am 24. Juni 2013 Enzalutamid unter dem Handelsnamen Xtan$\mathrm{di}^{\circledast}$ zur Behandlung von Männern mit metastasiertem kastrationsresistentem Prostatakarzinom, das während oder nach einer Chemotherapie mit Docetaxel fortschreitet, zugelassen. Die Zulassung basiert auf den Ergebnissen der Phase-III-Studie AFFIRM [Scher HI et al. J Clin Oncol. 2012;30(suppl 5):abstr. LBA1]. Danach führte die orale Einnahme des Androgenrezeptor-Signalweginhibitors Enzalutamid im Vergleich zur Placeboeinnahme zu einer statistisch signifikanten Verlängerung des Gesamtüberlebens von 4,8 Monaten $(\mathrm{p}<0,0001)$.

red

Nach Informationen von Astellas Pharma 